Review Article

\title{
Almis anterolateral hip approach using a different table and legs position during femoral exposure; new surgical technique
}

\begin{abstract}
One of best MIS hip approaches is at least theoretically the Röttinger's muscles sparing Watson-Jones anterolateral approach in decubitus lateralis but in our hands difficulties were present, especially during leaning curve, to mobilize the femur without excessive superomedial capsule and external rotators e.g. piriformis and/or obturator internus release. External rotators release increases hip laxity and longer arthroplasty necks are usually used resulting to leg lengthening. In this new approach only the gluteus minimus insertion tendon is temporary elevated. The operating leg is not placed in extension but in $20^{\circ}$ flexion, to avoid stress on abductors, as also in adduction and external rotation. The opposite leg is stabilized at the posterior leg support and not the anterior that is removed. The opposite leg on its support in slight abduction and extension and $15^{\circ}$ to $20^{\circ}$ anterior inclination of the table facilitates even more the femoral access in difficult cases without difficulties.
\end{abstract}

Volume 7 Issue 4 - 2017

Nikolaos Christodoulou

Chief Director of Orthopedic Department, Greece

\begin{abstract}
Correspondence: Nikolaos Christodoulou, Orthopedics surgeon, Chief Director of Orthopedic Department, Athens Medical Group-latriko Psychiko Clinic, Athens, Antersen I street, Neo Psychiko I I525, Greece, Tel 210-6974000 Email chnortho@yhoo.grn.christodoulou@iatriko.gr;www. christodoulou.n.gr
\end{abstract}

Received: January 29, 2017| Published: March 10, 2017

Keywords: Hip, MIS hip replacement, Anterolateral approach, Anterior approach, ALMIS approach, Non-invasive technique, New hip arthroplasty technique, Hip arthroplasty leg position

Abbreviations: MIS, Minimally Invasive Surgery; DA, Direct Anterior; ASI, Anterior Supine Intermuscular; DS, Direct Superior; DL, Direct Lateral

\section{Introduction}

Many different minimally invasive surgery (MIS) hip approaches e.g. using two incisions, Computer assisted, Direct Anterior (DA) or AMIS, Anterior Supine Intermuscular (ASI), Anterolateral MIS (ALMIS), Direct Superior (DS), Super-Path, Direct Lateral (DL) and so on, have been proposed last 15 years in order to reduce skin, muscles, nerves, vessels and bones injuries but clinically the perfect one is not yet found. The classic position of the operating leg during femoral exposure in lateral or anterolateral classic approaches in full flexion, adduction and external rotation (Figure 1) brings the gluteal abductor muscles on traction and possible injury. In modified by Heinz Röttinger's muscles sparing Watson-Jones anterolateral approach,,$^{1-3}$ which passes between tensor fascia latae and gluteus medius, the operating leg is placing in extension, adduction and external rotation during femoral access (Figure 2) in order to minimize this risk. Usually measurable abductors injury is avoided. ${ }^{4-6}$

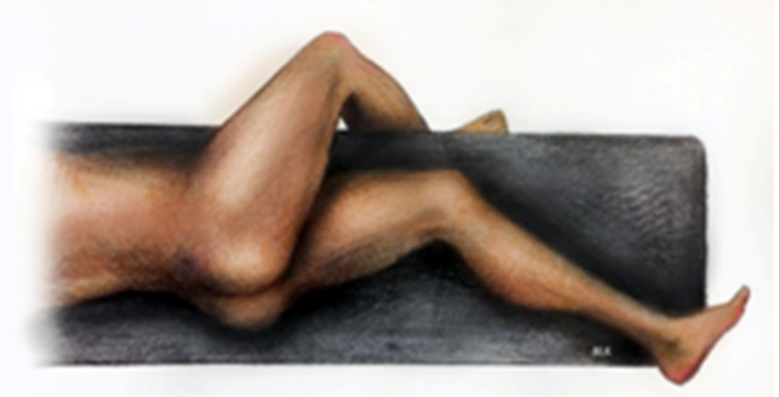

Figure I Classic lateral or anterolateral hip approaches; operating leg in full flexion, adduction and external rotation.

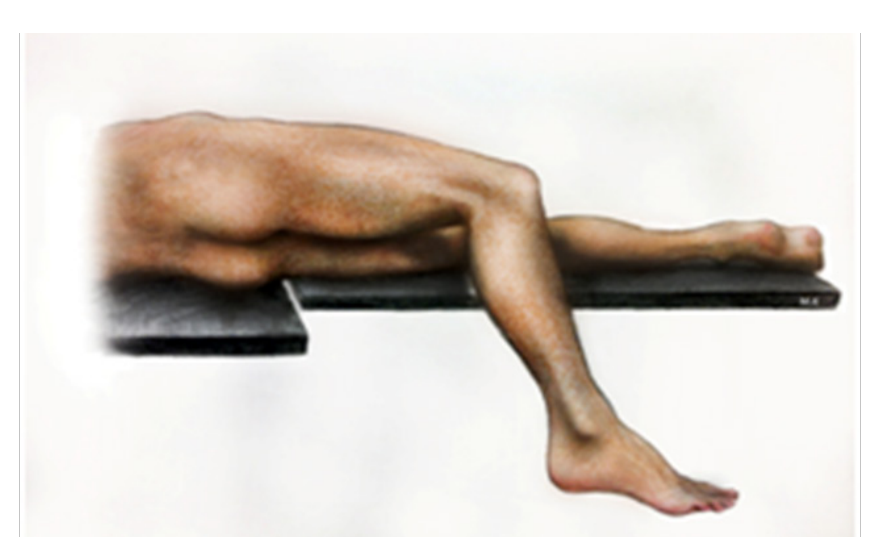

Figure 2 Rottinger type anterolateral approach; operating leg in extension, adduction and external rotation.

Trying to improve even more during last years our previous less invasive and bloodless lateral hip approach. ${ }^{7}$ in order to avoid even more the temporary elevation of the anterior insertion of the gluteus medius muscle we tested in about 20 cases the theoretically less invasive Rottinger's anterolateral approach (Figure 2) with the operating leg in extension but we had many difficulties during learning curve to mobilize the femur especially in obese patients without excessive supero-medial or posterior capsule and external rotators mainly piriformis or/and obturator internus release from inside, as it is also mentioned by others. ${ }^{1,8,9}$

Pflüger and collaborators (2007) report that shaft fissures occurred early in the development of the anterolateral in supine position MIS hip approach with the leg also extended during femoral exposure and led them to recommend routine capsular release, and when necessary, additional incision of the piriformis tendon .9

So, after two femoral shaft fractures in osteoporotic patients during the femoral access with the operating leg in extension we abandoned the Rottinger's or Pflüger's approaches. The excessive supero-medial 
capsule and external rotators release also in other three cases produced hip laxity and a longer arthroplasty neck was needed to stabilize the hip. This had the convenient of leg lengthening more than one $\mathrm{cm}$ in two cases and the need of offset stem in the other one resulting in trochanteric pain syndrome. In order to avoid these problems we use last four years a different table and legs position mainly during femoral access. The anterior and not the posterior leg table support is removed during surgery and the contralateral leg support is positioned in slight downloads and posterior tilting (Figure 3). This results to an easy and safe femoral access reducing gluteus medius traction or rasping injury. (Figure 4) A slight anterior tilting of the surgical table as a whole at about $15^{\circ}$ to $20^{\circ}$ facilitates even more the femoral preparation in difficult cases (Figure 5).

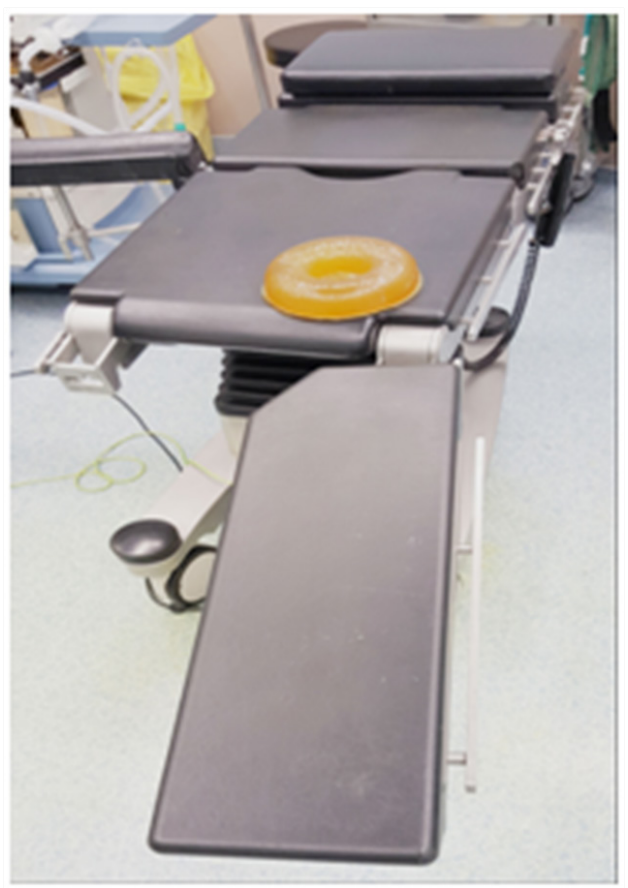

Figure 3 Table position; the anterior leg support is removed and the contralateral leg support is tilting downwards and outwardly.

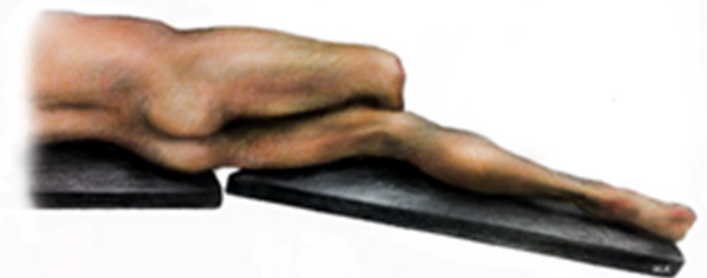

Figure 4 Patient and legs position in the modified new ALMIS hip approach.

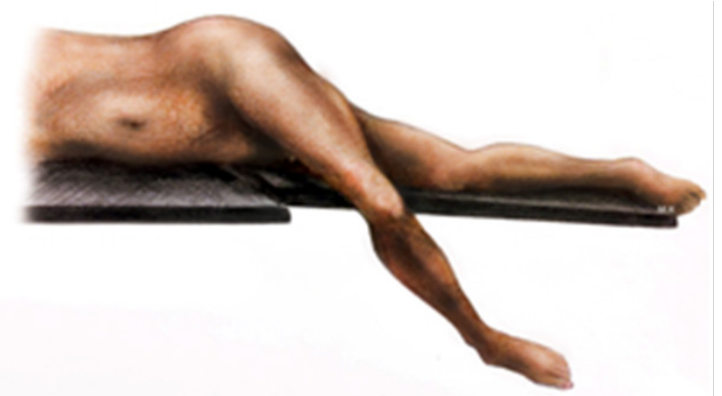

Figure 5 Leg position in slight flexion, adduction, external rotation at the region of the removed anterior leg support accompanied by slight anterior tilting of the table during femoral exposure and reaming in difficult cases.
Only a temporary minimal and full reparable muscle injury has been scheduled to happen in this new approach and is the elevation of gluteus minimus insertion tendon from greater trochanter and capsule accompanied by some mechanically not important anterior medius gluteus fibers. The tendon is strongly resutured at the end of the surgery at its normal place without consequences. It must be mentioned that the gluteus minimus is injuried in many theoretically atraumatic anterior, lateral or posterior MIS hip approaches but as is in contact with the capsule and is displaced proximally under the gluteus medius this injury is often ignored (10-12).

Gluteus minimus seems to have a minor impact on the clinical outcome particularly not on the abduction strength. ${ }^{5}$

\section{Anesthesia}

Anesthesia may be subarachnoid spine block, epidural or general anesthesia. Only dorsal-subarachnoid anesthesia is used in the majority of our patients.

\section{Patient and table positioning}

The patient is positioned in lateral decubitus position slight dorsally and the contralateral greater trochanter, on which the patient is lying, as close is possible to the edge of the table. (Figure 6).

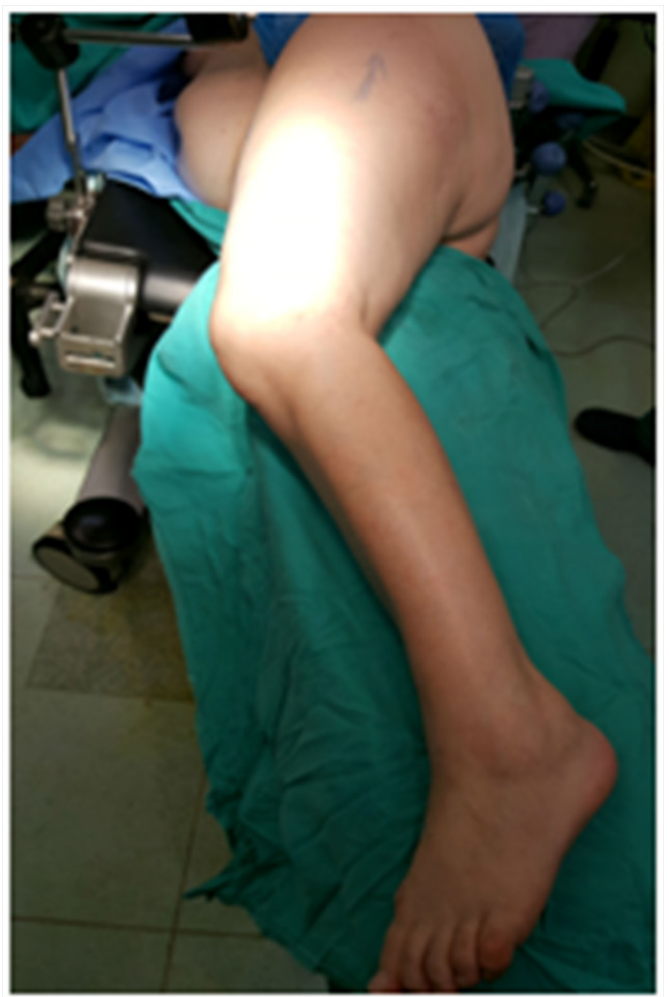

Figure 6 Patient positioning.

Ideally the patient is positioned on a pediatric vacuum matress or an elastic gelly cyclic pillow is placed under the contralateral greater trochanteric region in order to stabilize the pelvis and minimize the skin pressure during surgery (Figure 3 ). Contralateral leg is stabilized on the posterior leg support with a belt or elastic bandaging at the level of the knee joint. Few degrees of extension about 10 to 20 degrees and slight abduction of the contralateral leg with its support as a whole is useful for better frontal mobilization of the operated leg during femoral access mainly in difficult cases e.g. obese patients. 
The hip is prepared and draped using contemporary techniques which allow to the affected leg to be draped free and to be mobile in front of the table during the surgical procedure.

\section{Anatomical landmarks and incision}

The operating leg position during access to the hip joint is neutra in slight external rotation. The incision is normally longitudinal at the greater trochanter region of about 6 to $11 \mathrm{~cm}$ (mean $8 \mathrm{~cm}$ ) in length depending on the thigh perimeter and the peculiarities of each patient with $1 / 2$ proximally and $1 / 2$ distally of the tip of greater trochanter (Figure 7). It can easily extended proximally and distally (Figure 8) in obese patients or other difficult cases as dysplasia or revision surgery without vessels or nerves problems e.g. lateral femoral cutaneous nerve or deep femoral artery branches which impose some restrictions at the anterior MIS approaches.

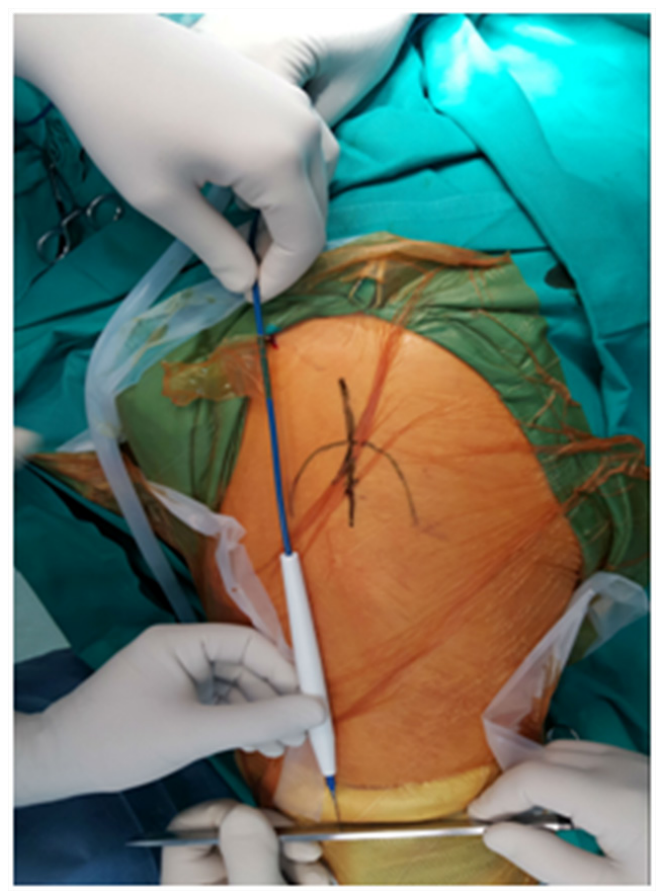

Figure 7 Skin incision marking and leg length differences measurement before surgery and after trial reduction using a small $4 \mathrm{~mm}$ in diameter external fixator pin at the iliac crest and a skin marker at the distal femoral region.

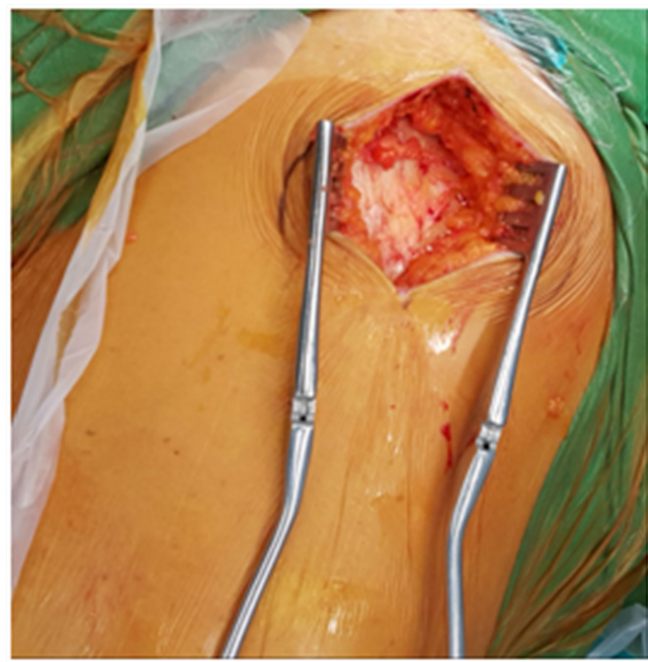

Figure $\mathbf{8}$ Lateral longitudinal skin incision at the greater trochanter region.

\section{Exploration for the intermuscular space}

The split of the aponevrosis of the tensor fascia latae is curved directed proximally to anterior iliac spine and distally to the longitudinal axis of the femur, 6 to $7 \mathrm{~cm}$ proximally and distally from the level of the greater trochanter (Figure 9). The top of the aponevrosis curve opening is located near to the posterior border of the greater trochanter in order to have excellent view of gluteus medius and avoid immediate contact of gluteus minimus and tensor fascia latae suture at the end of the surgery as also adhesions problems. An automatic soft tissue retractor is used to displace gluteus maximus and tensor fascia latae muscles. The interval between tensor fascia latae, gluteus medius and vastus lateralis is exposed. The gluteus minimus tendon is found under a few elevated anterior fibers of gluteus medius, usually adipose and mechanically not important for abduction (Figure 11). The gluteus minimus tendon is shifted backwards and is hold by stitches to be controlled during surgery (Figure 11).

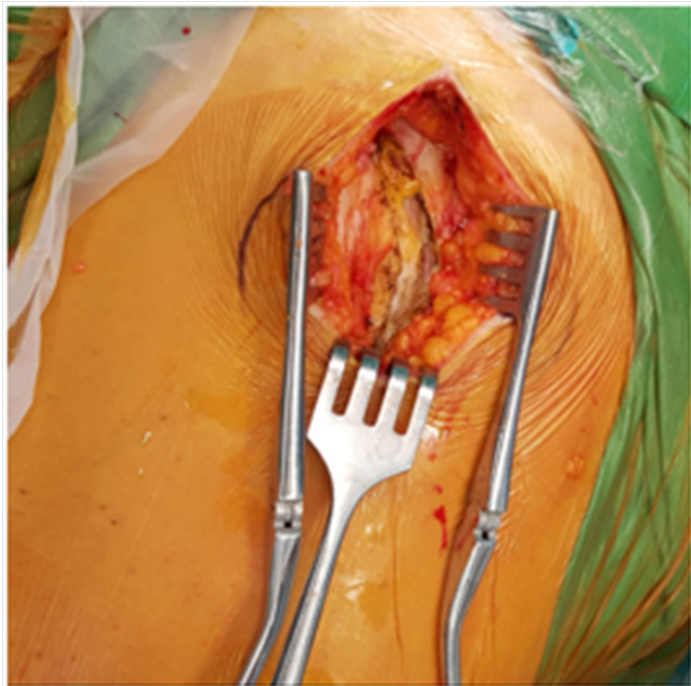

Figure 9 Fascia latae curved incision.

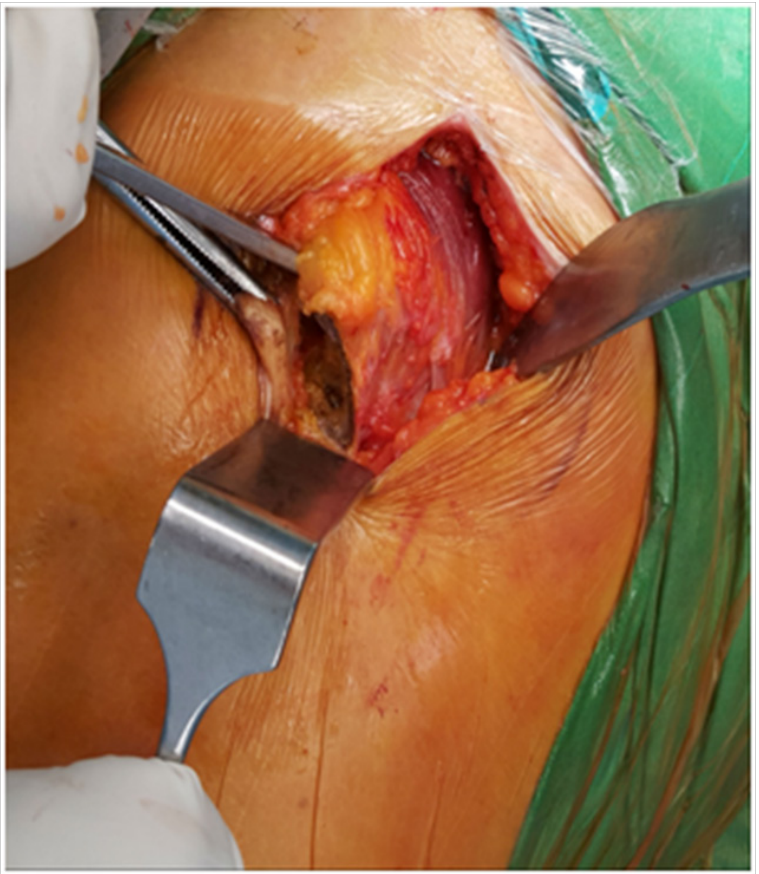

Figure 10 Elevation of the usually adipose and mechanically not important anterior gluteus medius fibers. 


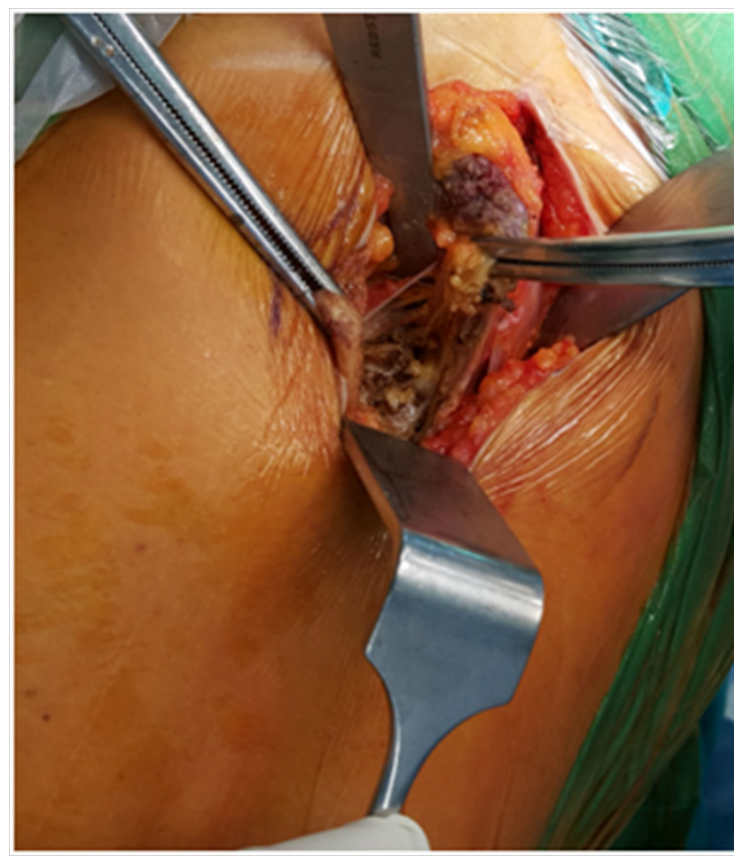

Figure I I Temporary elevation of gluteus minimus tendon.

In difficult cases e.g. dysplasia, revision cases or use of straight stems a small part of the gluteus medius anterior oblique insertion, about one to two $\mathrm{cm}$ in width, is also temporary elevated from greater trochanter and is resutured carefully during wound closure with the elevated gluteus minimus tendon as a whole at their normal place using strong Vicryl stiches. Usually no any serious vessel branch is at risk at this MIS approach if manipulations are detailed and careful. No visible anterior or posterior circumflex artery branches are found at the field of this approach. Electrocoagulation of very small venous plexus running along the anterior side of the gluteus medius rarely is needed. So this approach is simple, safe and excessively bloodless.

\section{Capsule exposure and arthrotomy}

Limb position: neutral, on or without leg support, in slight external rotation.

Finger palpation clearly identifies the extracapsular superior aspect of the femoral neck guiding the retractors positioning. A wide Cobra retractor is positioned on the superior border of the neck, between capsule and gluteals and parallel to their fibers direction and a second one on the antero-superior edge of the acetabulum under the indirect head of the rectus femoris. A reversed " $\mathrm{T}$ " or perpendicular " $\mathrm{H}$ " capsulotomy is performed. The capsular incision is extended up to the edge of the acetabulum and along the edge, somewhat anteriorly and posteriorly. Additional thin hohmann retractors, if needed, are also carefully used to remove roof osteophytes and to expose even more the femoral neck. The incision at the base of the greater trochanter is not extended to vastus lateralis fibers (Figure 12).

\section{Femoral neck osteotomy and femoral head extraction}

The dislocation is almost totally achieved by careful traction and progressive external rotation of the femoral head using a screwdriver handle (tirebusson) inserted to the usually sclerotic anterolateral femoral neck-head junction with all retractors removed for muscles relaxation. (Figure 12) The excessive and sudden traction, flexion and external rotation of the femur by the assistant surgeon to dislocate the hip must be avoided because rotational femoral fracture may occur in osteoporotic patients and injury on the gluteus medius and minimus muscles.

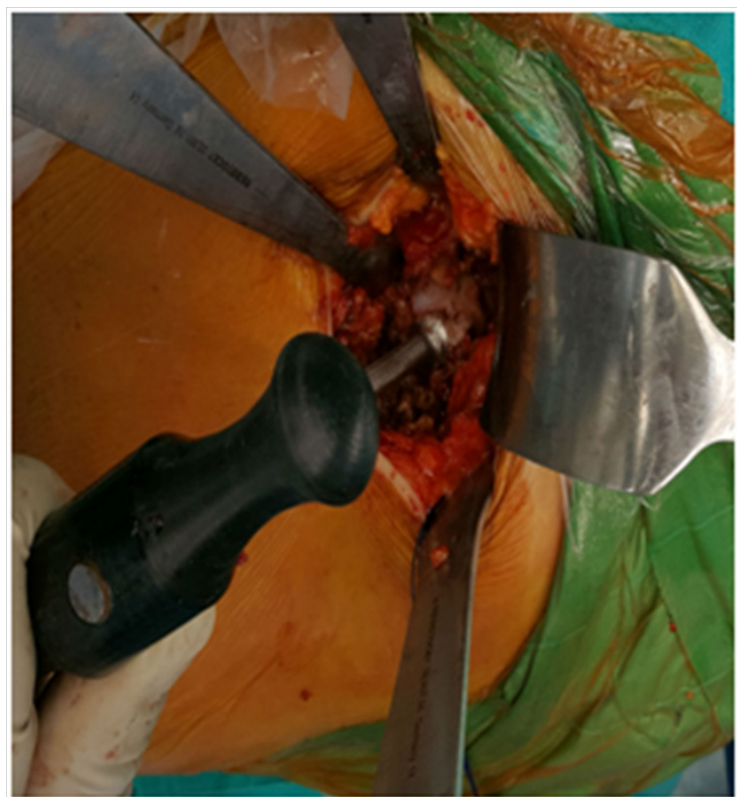

Figure 12 Femoral dislocation using careful traction and external rotation by a screwdriver handle (tirebusson) inserted to the usually sclerotic anterolateral femoral neck-head junction.

Femoral neck double osteotomy before head removal is normally avoided because is hemorrhagic, time consuming and sometimes is accompanied by fragmentation of the femoral head.

It is only used in cases in which the dislocation is not achieved with very slight and carful traction of the femoral head by the screwdriver handle and in some difficult protrusion acetabulum cases.

The dislocated neck is cut at the level determined by preoperative planning usually at an angle of approximately $45^{\circ}$ relative to the long axis of the femur and at an antevertion angle at about 10 to 15 degrees (Figure 13).

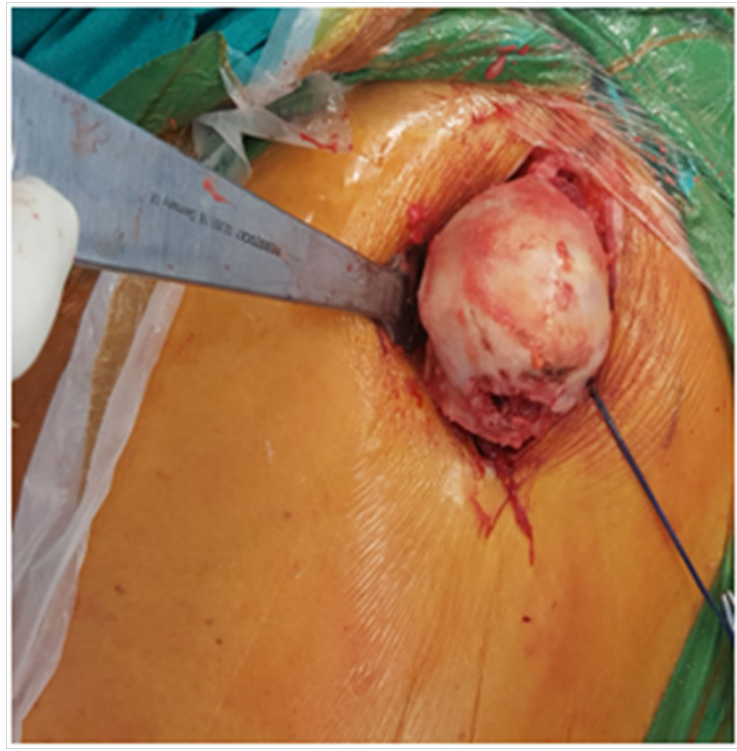

Figure 13 Extraction of the osteotomised femoral head.

This level of the osteotomy usually corresponds to distal limits of the hohmann's retractor posed at the "saddle" of the femoral neck. 
Haemostatic wax is temporary placed at the osteotomised femoral neck to eliminate the femoral neck bleeding during acetabulum preparation.

\section{Acetabulum exposure}

Limb position: neutral, on leg or without support, in slight external rotation.

An external fixator pin $6 \mathrm{~mm}$ at diameter is temporary inserted in difficult cases about 1 to $1.2 \mathrm{~cm}$ over the anterolateral acetabular ring at about 2 o'clock (or 10 o'clock, depending of the side) to protect tensor fascia latae muscle during cup preparation used also as a iliac bone point marker for checking the upwards cup placement (Figure 14).

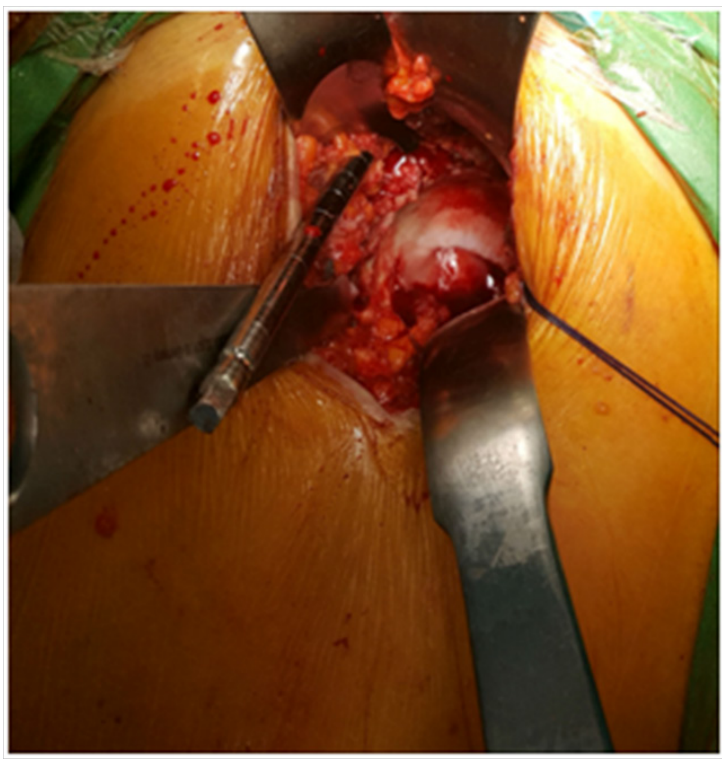

Figure I 4 External fixator pin of $6 \mathrm{~mm}$ in diameter temporary inserted about I to $1.2 \mathrm{~cm}$ over the anterolateral acetabular ring to retract tensor fascia latae used also as an iliac bone point marker for cup placing.

Three MIS Cobra type retractors are also placed to observe and prepare the acetabulum. One of moderate wide is positioned at about 7 o'clock (or 5 o'clock, depending on the side) to decline the femur backwards; a second wide is carefully placed without pressure under gluteals muscles and parallel to their anterior border at about 10 o'clock ( 2 o'clock). The third one is positioned at about 5 o'clock (7 o'clock) on anterior acetabular ring displacing tensor fascia latae muscle. Sometimes a double-spike Cobra retractor may be positioned near 4 o'clock ( 8 o'clock) at the sides of the acetabular notch instead of the simple one posed at 5 o'clock ( 7 o'clock). A possible acetabular synovial fold at this region is previously cut. All of the procedures of capsular preparation, osteophyte resection and acetabular preparation are performed in the usual manner (Figure 15). An offset reamer handle is not usually necessary and only in difficult cases eccentric MIS reamers are used to facilitate cup preparation.

Careful distal traction on the femur using a hook retractor may help the acetabulum exposure in very difficult cases and cup positioning (Figure 16).

The acetabular metal back and cup insert are positioned purely according to the anatomic landmarks taking into consideration the possible changes of anterior pelvis tilting from the diminution of the lumbar spine lordosis in decubitus lateralis position or fixed flexion deformity of the contralateral hip joint (Figure17).

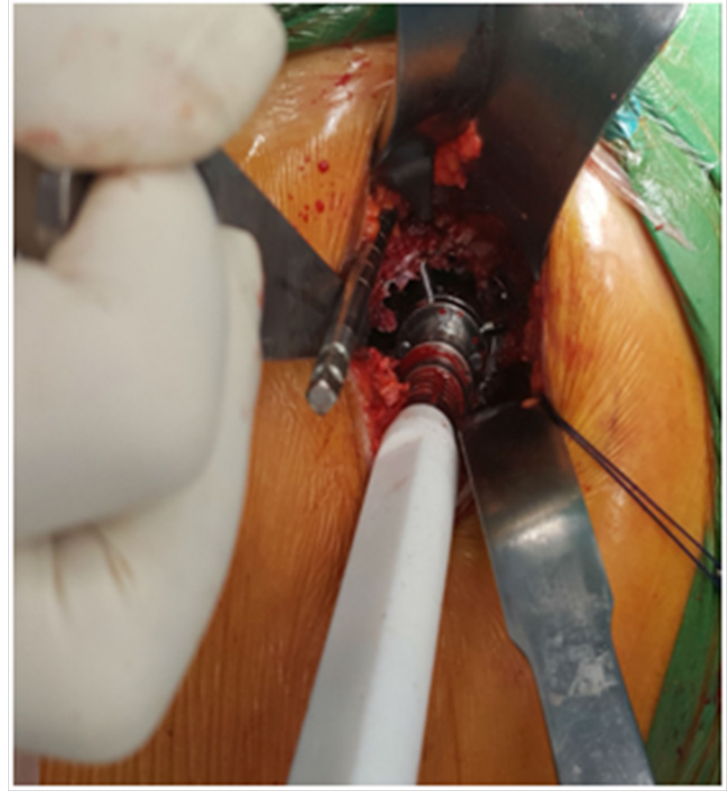

Figure I5 Acetabulum reaming.

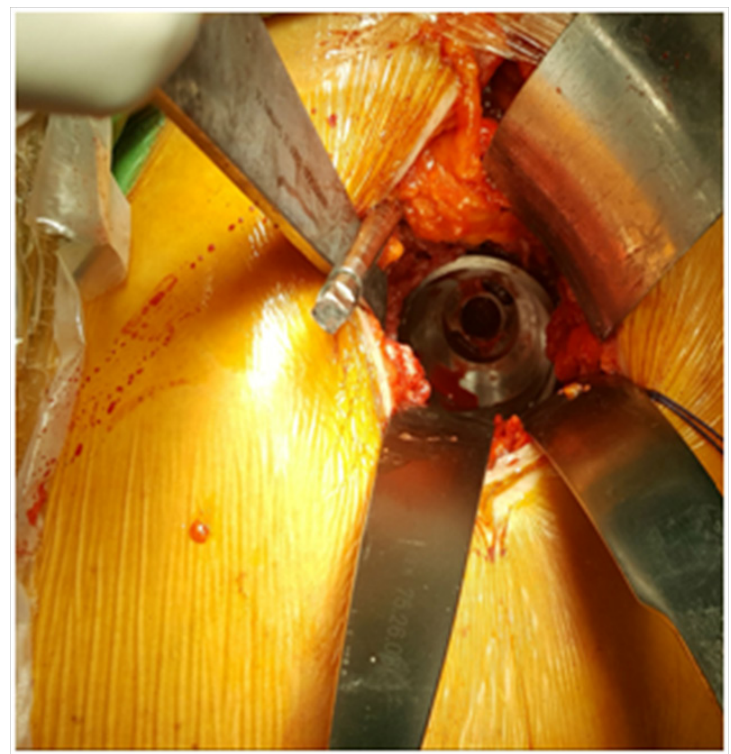

Figure 16 Cup metal back positioning.

Usually modern biologically fixed Zweymuller or similar type new generation threaded cups are usually used. Their special lamellae stabilize steadily the cup mainly at the columns region without any need of roof screws. The main indication of these cups is inadequate acetabular roof cases. ${ }^{3}$

\section{Femoral exposure - new leg position}

The table and leg position during femoral exposure is different from that usually used in classic lateral or anterolateral approaches (full flexion, adduction, external rotation) or in Röttinger type anterolateral approach (Extension, adduction, external rotation).

The lower limb is positioned during femoral exposure in less than $20^{\circ}$ flexion, $20^{\circ}$ adduction and $90^{\circ}$ external rotation in front of the table, over the contralateral knee joint, and at the place of removed anterior leg support. The contralateral leg with its support as a whole 
is positioned in slight extension and abduction for better frontal movements of the operating leg (Figure 4).

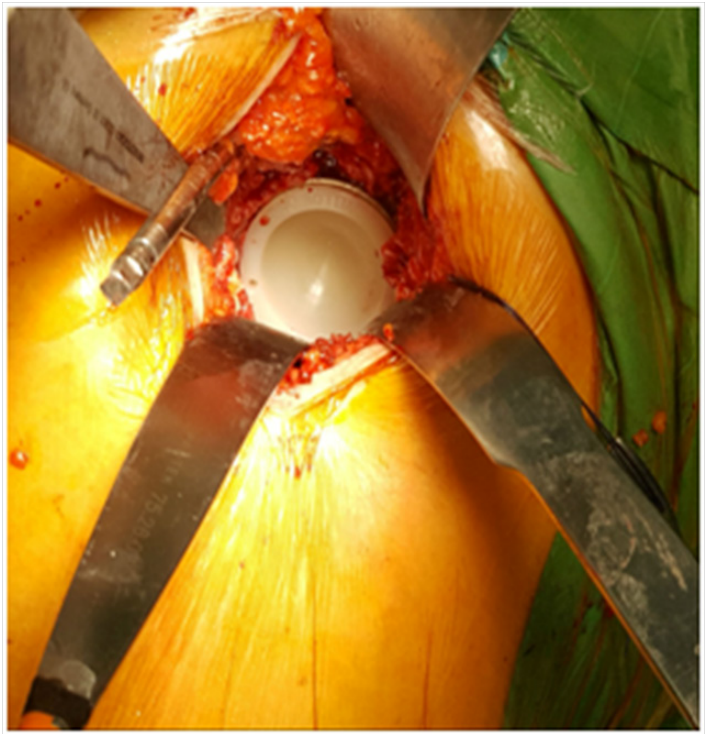

Figure 17 Cup insert positioning.

Additionally slight temporary anterior tilting of the table facilitates even more the femoral exposure and preparation (Figure 5). So, there is no need of supero-medial or posterior thick capsule or external rotators release e.g. piriformis and/or obturator internus to mobilize the femur except in some dysplasia or low congenital hip dislocation cases in which leg lengthening is needed.

It is preferable to use curve rasps with or without eccentric rasp machine assistance as also curve stems, short or long depending of age and bone quality, to avoid even greater trochanter or abductors rasping injury (Figure 18).

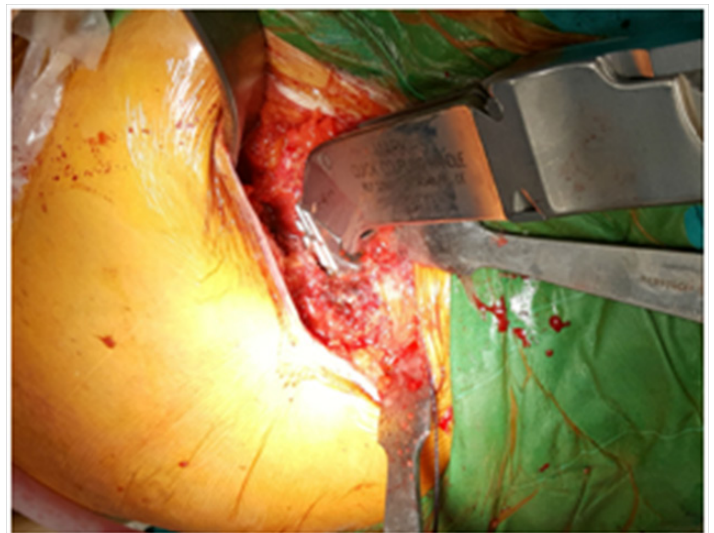

Figure I 8 Easy and safe intramedullary femoral canal reaming without any need of capsular or external rotators release.

\section{Implant fixation}

The positioning of the acetabular and femoral components is assessed as usual. The cup normally is placed immediately after acetabular preparation. Using trial femoral stem and head after femoral rasping the hip is reduced and range of motion, legs length equality and no impingement are confirmed (Figure 19). Fixation may be performed with or without cement. Normally we use Zweymuller or similar type new generation biologically fixed modern threaded cups which have special lamellae in order to be fixed steadily not only at the acetabular roof but also well at the columns region where screws are impossible to be used. The main indication is the inadequate acetabulum roof cases. ${ }^{13}$ The stems are also biologically fixed antirotation type of rectangular section. The curve type antirotation stems, short or long, are preferable than the straight ones. In younger patients and good quality bone stock as we have seen modern short curved stems have shown excellent early results (Figure 20).

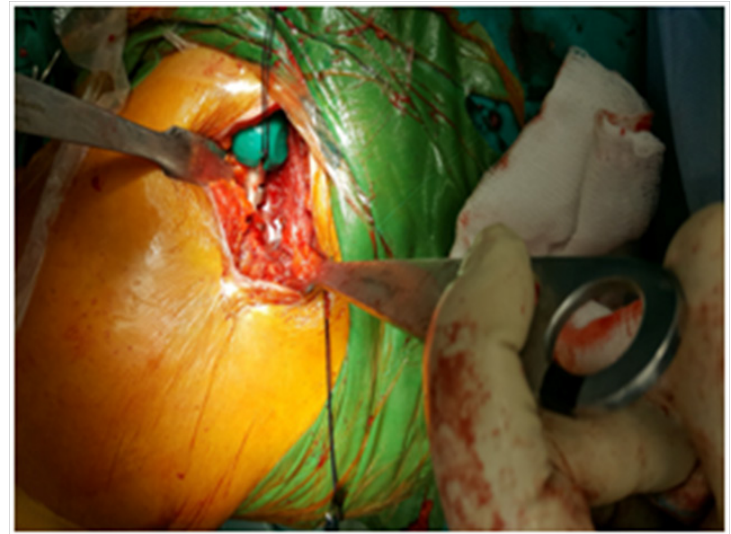

Figure 19 Trial femoral stem and head implants for reduction, stability and leg length control.

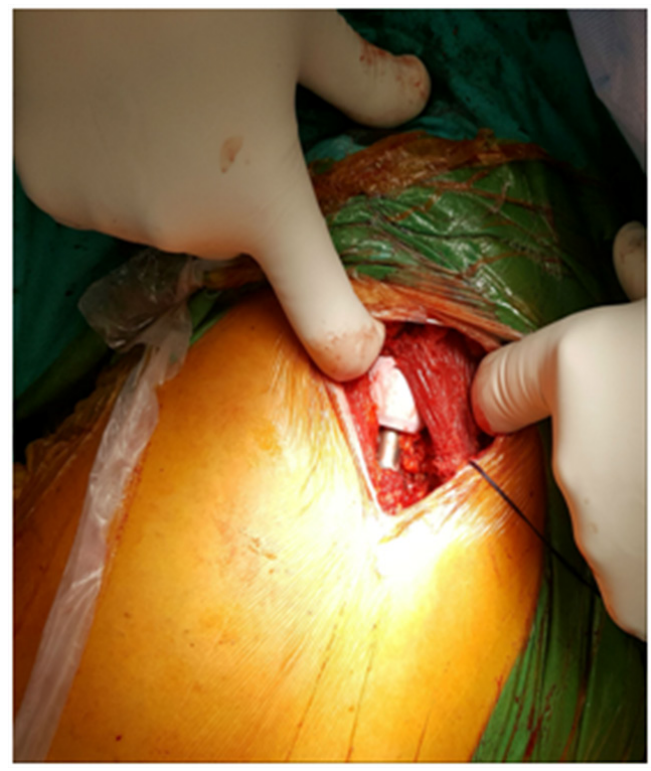

Figure 20 Final arthroplasty stem and head implants. The intact gluteus medius muscle is also.

Their placement is easy, no traumatic in the distal intramedullary canal and also much periprothetic bone stock is remaining for any possible revision case. The early results are very promising. Only two mechanical loosening during learning curve of a total of 142 cases were found in 6 months to 3 years follow-up. In difficult high congenital dislocation cases other hip approaches may be used.

\section{Closure}

Almost the entire and important gluteus medius muscle mass remains intact at the end of the surgery (Figure 21). So, in correlation with the absence of external rotators and posterior capsule release free early hip motion is possible in all directions (Figure 22). The avoidance of supero-medial and posterior capsule or external rotators release minimizes the dislocation probability so the remaining capsule is not closed. The gluteus minimus tendon which was temporary transposioned backwards under the gluteus medius is reinserted using strong Vicryl stitches near to its normal position at the greater 
trochanter. It is accompanied by the few anterior temporary elevated fibers of medius gluteus muscle as a whole. An additional crosswise like eight suture between anterior gluteals insertion and vastus lateralis aponeurosis offers even better restraining. The hemostasis in the venous plexus anterior to the gluteus medius muscle rarely is needed. The fascia lata, subcutaneous tissues and skin are sutured in place. The different suture region of gluteus minimus in frond and the fascia latae backwards probably helps as we have seen in our patients to the diminution of soft tissue impingement or adhesions at the greater trochanter region. Bioabsorbable stitches are usually used for skin closure (Figure 23).

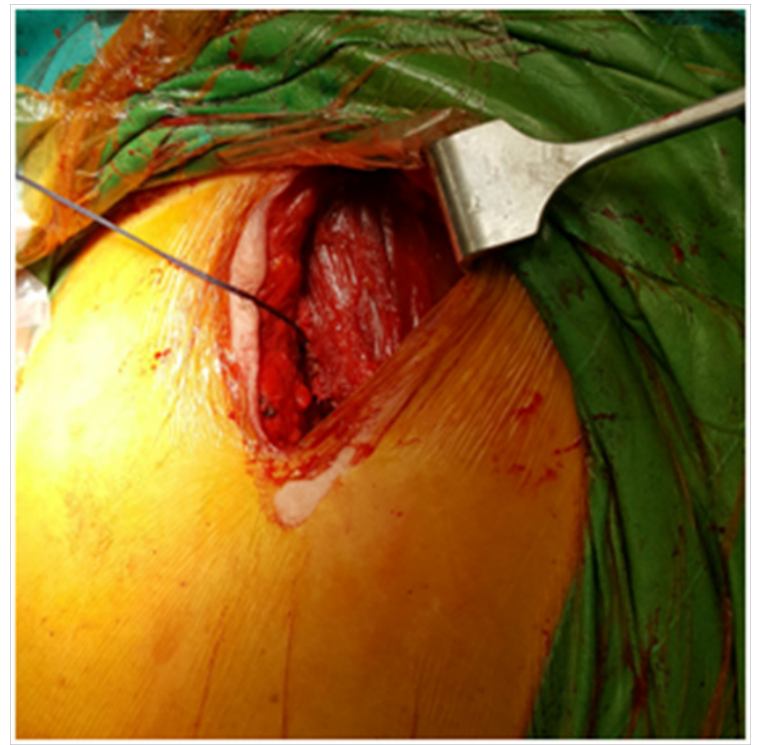

Figure 2I Almost the entire and important gluteus medius muscle mass remains intact at the end of the surgery.

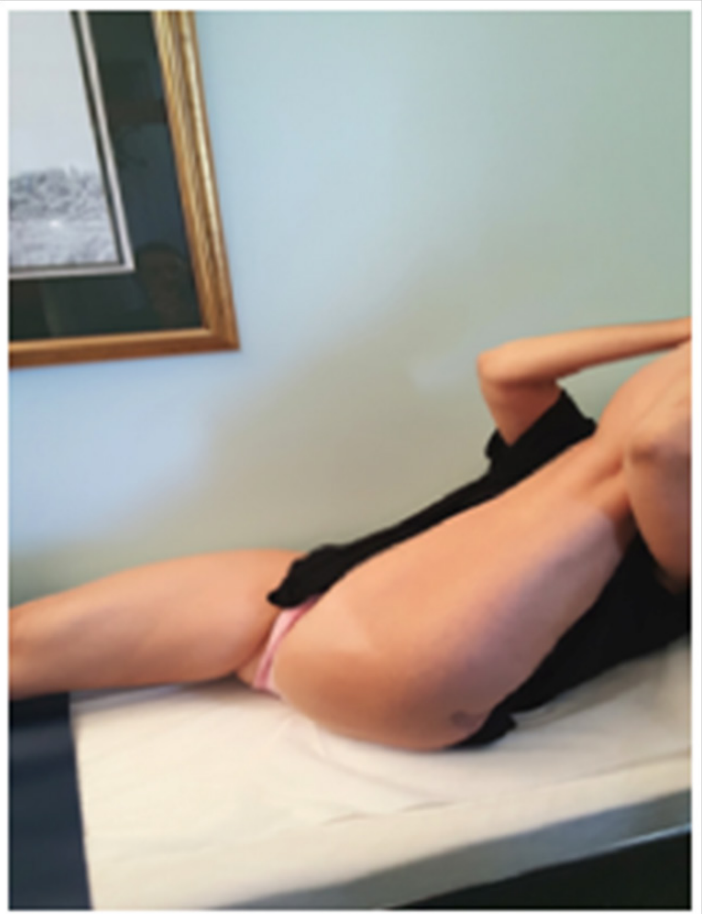

Figure 22 Excellent hip stability and full free hip motion is possible in many cases one month after surgery.

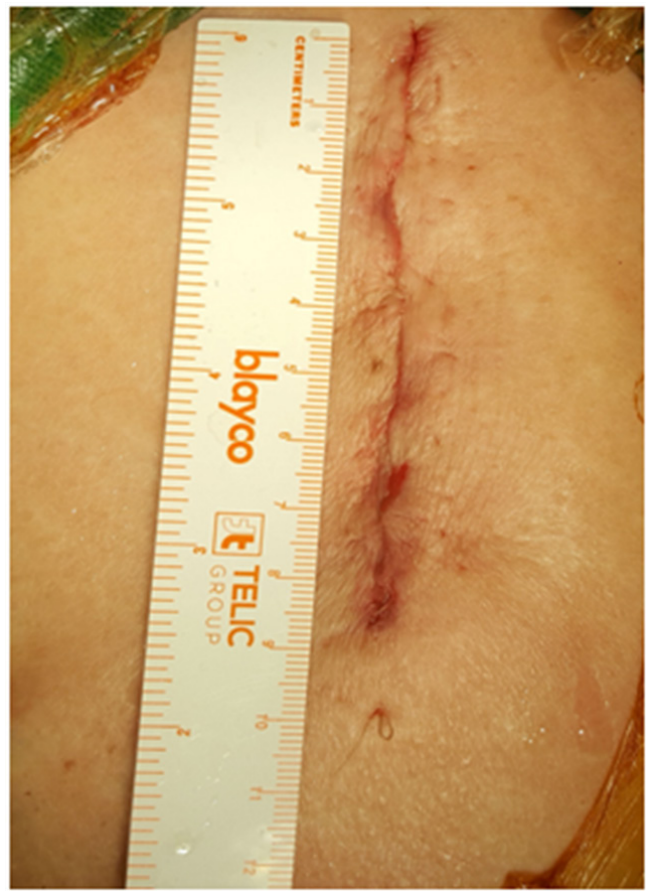

Figure 23 Skin closure by using bioabsorbable stitches.

\section{Conclusion}

This new proposed modified anterolateral MIS hip approach is not only minimally invasive in gluteal muscles but also sparing in all serious anatomical elements of the hip as are the supero-medial or the posterior capsule and the external rotators which are often released from inside..$^{911}$ in the anterior or anterolateral MIS approaches or from outside in almost all posterior approaches to mobilize the femur. This is mainly due to the special patient and surgical table or leg support position of this new method. The operating leg is positioned during femoral exposure in slight flexion, adduction and external rotation instead of extension, adduction and external rotation of Röttinger's anterolateral hip approach. Another difference from Röttinger's approach is that during surgery and with the patient at decubitus lateralis position the anterior leg support is removed instead of the posterior one and the opposite leg is stabilized on the remaining posterior leg support. The contralateral leg and its support as whole is lightly extended and abducted to facilitate the free movements of the operating leg.

In very difficult cases, during femoral preparation, a slight anterior tilting of surgical table as a whole facilitates even more the femoral preparation.

Only the gluteus minimus insertion tendon is temporary elevated and shifted backwards hold by stitches during surgery. The tendon is resutured at the end of the operation at its normal position accompanied in some cases with the mechanically not important anterior fibers of gluteus medius muscle. This simple process facilitates the femoral access and minimizes the gluteus minimus and gluteus medius main muscle mass injury by traction or manipulations during surgery and also protects by displacing proximally the superior gluteal nerve anterior branches. This gluteus minimus tendon temporary injury is not important. This minimal injury of the gluteus minimus usually has a minor impact on the clinical outcome particularly not on the abduction strength. ${ }^{4-6,12}$, The main function of the gluteus minimus is rather the centralization of the femoral head in the joint during the gait 
cycle and participation in internal hip rotation than hip abduction and stabilization of the pelvis. ${ }^{5}$ This is sufficiently ensured by strong suture of gluteus minimus tendon at its normal place, so the consequence is not serious. In contrary the posterior or supero-medial capsular excessive release accompanied with external rotators release in order to mobilize the femur during its exposure and intramedullary canal reaming necessary in other MIS type anterior or anterolateral hip approaches with the leg in extension has according to our experience the result of joint laxity in some cases. So, in order to stabilize the hip after trial reduction in these cases a longer than normal stem neck is usually needed resulting to leg lengthening or offset stem use accompanied by trochanteric pain syndrome.

Excessive capsule or external rotators release are necessary only in cases in which leg lengthening is needed as are dysplasia, low congenital dislocation or stiff posttraumatic cases. This approach may be used systematically in all primary (Figure 24) or secondary osteoarthritis even in dysplasia or low congenital dislocation cases as also in obese patients. In these cases, the skin incision may easily be extended without e.g. lateral cutaneous nerve of the thigh or deep femoral artery branches limitations of the anterior MIS approaches. The main restriction is a previous posterior unsuccessful arthroplasty with presence of many materials and severe posterior wall insufficiency. Course in 525 patients operated with this new technique, in 142 of them using a short curved uncemented stem (Figure 25), is spectacular compared to the classic approaches and more successful than our previously published less invasive and bloodless lateral MIS approach.

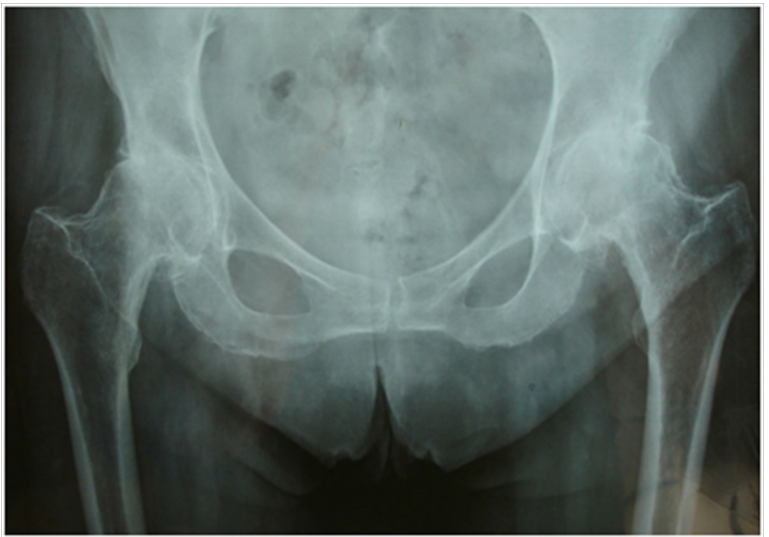

Figure 24 Preoperative X-ray. Osteoarthritis of both hip joints, mainly of left side.

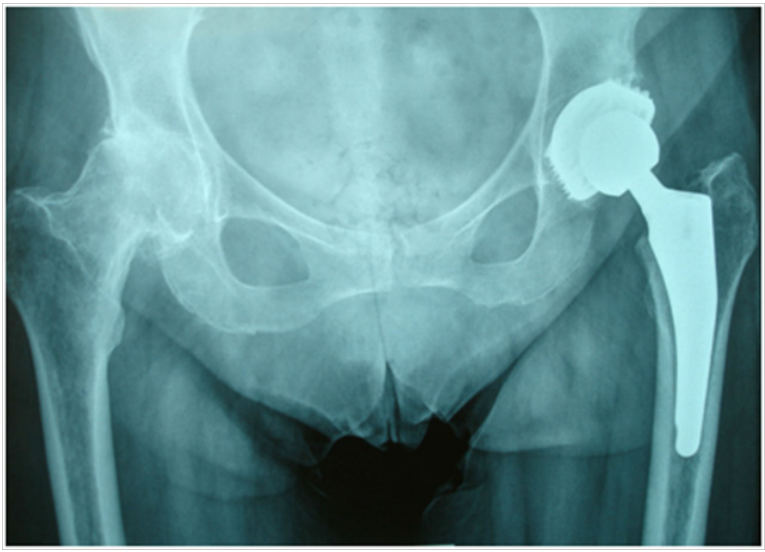

Figure 25 Post-operative X-ray.A threaded biologically fixed cup and a short curved antirotation uncemented stem were used at left side.

\section{Acknowledgements}

I have the obligation to express my acknowledgements to the very specialized in orthopedic patients anesthetist Korre Marianna, to my colleagues orthopedic surgeons Voutsas Akis, Baxevanos Nikolaos, Volonakis Emmanouil and Renieri Natalia as also to the secretaries of the Clinic Sirigou Aggeliki, El Azab Katerina and katirzoglou Alina.

\section{Conflicts of interest}

None.

\section{References}

1. Aebi J, Giraud M Non invasive modified anterolateral approach in total hip arthroplasty. Orthopaedics \& Traumatology: Surgery \& Research. 2011;97(6):675-680

2. Bertin KC, Röttinger H Antero-lateral mini-incision hip replacement surgery. A modified Watson-Jones approach. Clin Orthop Rel Res. 2004;429:248-255.

3. Hansen BJ, Hallows RK, Kelley SS The Rottinger approach for total hip arthroplasty: technique and review of the literature. Curr Rev Musculoskelet Med. 2011;4(3):132-138.

4. Muller M, Totz S, Dewey M et al. Evidence of reduced muscle trauma through a minimally invasive antero-lateral approach by means of MRI. Clin Orthop Relat Res. 2010;468(12):3192-3200.

5. Muller M, Tohtz S, Winkler T et al. MRI findings of gluteus minimus muscle damage in primary total hip arthroplasty and the influence on clinical outcome. Arch Orthop Trauma Surg. 2010;130(7):927-935.

6. Jixiang $\mathrm{T}$, Hong $\mathrm{C}$, Cheng $\mathrm{C}$ et al. The strength and function of hip abductors following anterolateral minimally invasive total hip arthroplasty. Chinese Journal of Traumatology. 2014;17(2):73-78.

7. Christodoulou N, Dialetis K, Gouzias G et al. Modified less invasive and bloodless lateral hip approach for total arthroplasty. European Journal of Orthopaedic Surgery \& Traumatology. 2012;22(2):167-174.

8. Matsuura M, Ohashi H, Okamoto Y et al. Elevation of the Femur in THA Through a Direct Anterior Approach: Cadaver and Clinical Studies. Clin Orthop Relat Res. 2010;468(12):3201-3206.

9. Pflüger G, Junk-Jantsch S, Schöll V Minimally invasive total hip replacement via the anterolateral approach in the supine position. Int Orthop. 2007;31(Suppl 1):7-11.

10. Lüdemann M, Kreutner J, Haddad D et al. MRI-based measurement of muscle damage after minimally invasive hip arthroplasty. Der Orthopade 2012;41(5):346-353.

11. Meneghini RM, Pagnano MW, Trousdale RT et al. Muscle Damage During MIS Total Hip Arthroplasty: Smith-Peterson versus Posterior Approach. Clin Orthop Relat Res. 2006;453:293-298.

12. Parratte S, Pagnano MW Muscle damage during minimally invasive total hip arthroplasty: cadaver-based evidence that it is significant. Instr Course Lect. 2008;57:231-234.

13. Christodoulou N, Dialetis K, Christodoulou A High hip center technique using a biconical threaded Zweymuller cup in osteoarthritis secondary to congenital hip disease. Clin Orthop Rel Res. 2010;468(7):1912-1919. 